\title{
Modified keyhole technique for the treatment of biceps long head tendon rupture
}

\author{
YAO HUANG, HAO SHU, BING HE, FUCHENG ZHANG, \\ CHUNSHENG ZHANG, CHENGJIAN PENG and LUNING SUN
}

\begin{abstract}
Department of Orthopedics, Sports Medicine Center, Affiliated Hospital of Nanjing University of Chinese Medicine, Nanjing, Jiangsu 210029, P.R. China
\end{abstract}

Received January 13, 2019; Accepted September 10, 2019

DOI: $10.3892 /$ etm.2019.8053

\begin{abstract}
The present study aimed to assess a novel modified keyhole fixation technique to treat long head of biceps (LHB) rupture. From May 2015 to July 2017, a total of 9 patients with LHB rupture, decreased muscle strength and pop-eye sign were treated using the keyhole fixation technique combined with extramedullary cortical bone microplate-suspending fixation. Measurements of visual analog scale (VAS) score, elbow joint rotation, flexion strength, the Shoulder Score of the University of California Los Angeles (UCLA) and the Rating Scale of American Shoulder Elbow Surgeons (ASES) score were used to evaluate surgical outcomes prior to and following surgery. The 9 patients with LHB rupture included in the study were followed up for one year post-surgery. The supination and flexion elbow strength of all patients after 1 year was grade $\mathrm{V}$ and shoulder pain was relieved. The VAS, UCLA shoulder and ASES scores at one year post-surgery were significantly improved compared with those prior to surgery. In conclusion, modified keyhole fixation for LHB rupture features low difficulty of operation, high safety and reliable fixation, and is therefore a promising novel technique for the treatment of LHB rupture.
\end{abstract}

\section{Introduction}

The long head of the biceps (LHB) enters the shoulder joint at a large angle and its lesion is a common cause of shoulder pain (1). LHB lesions include tendinitis, tendon wear, superior labral tear from anterior to posterior (SLAP) lesion and partial or complete rupture (2). Isolated LHB lesions are rare and are frequently accompanied by rotator cuff tear or acromial impingement (3). Tendon fixation is a reliable and effective surgical procedure for

Correspondence to: Dr Luning Sun, Department of Orthopedics, Sports Medicine Center, Affiliated Hospital of Nanjing University of Chinese Medicine, 155 Hanzhong Road, Nanjing, Jiangsu 210029, P.R. China

E-mail: slnsportsmedicine@163.com

Key words: long head of biceps, rupture, fixation, keyhole, endobutton the treatment of LHB lesions (4). A number of surgical methods have been used in the treatment of LHB, including open, small incision or complete arthroscopy surgery, with a variety of fixing methods, including high or low tenodesis, bone tunnel fixation, soft tissue fixation, keyhole fixation, as well as anchor or interference screw fixation (5). Siebenlist et al (6) demonstrated a novel fixation method to treat LHB involving suspension from microplates on the inner surface of the cortical bone, close to the humerus. This fixation technique has resulted in good short-term biomechanical results; however, this relies solely on the fixation on a single cortical bone. The tendon is in contact with a small area of the cortical bone surface, and this may be detrimental to tendon-bone healing. This technique also requires pulling of the guide pin through the contralateral side to pull out soft tissue, which may cause axillary nerve injury. In this light, the present study provides improvements to the technique and counteracts these problems associated with the original treatment. Combined with keyhole technology, the intramedullary microplate was transferred to the surface of the ipsilateral cortical bone, and this treatment was used in 9 patients with LHB ruptures, pop-eye sign and decreased muscle strength. Application of this method resulted in beneficial short-term effects.

\section{Patients and methods}

Patient information. Between April 2015 and July 2017, LH modified keyhole suspension fixation was performed on 9 patients with LHB rupture exhibiting pop-eye sign and decreased flexion strength. The cohort included 8 males and 1 female aged between 43 and 65 years of age, and all were physical laborers. In all patients, the affected limb was that on the right side. A total of 6 patients complained of shoulder pain after carrying heavy objects, which led them to visit the hospital. A total of 3 patients had long-term shoulder pain and discovered pop-eye sign at the right upper extremity without any obvious cause. All patients were aware that they exhibited a decline in elbow strength. The study inclusion criterion was isolated LHB rupture without surgical contraindications, while the exclusion criteria were surgical contraindications. Each patient provided written informed consent. All of the procedures performed in the present study complied with the ethical requirements of the Affiliated Hospital of Nanjing University of Chinese Medicine (Nanjing, China). 
Surgical technique. Surgery was performed under general anesthesia. Each patient was placed in the beach chair position and the affected limb was placed at an abduction of $20^{\circ}$ and elbow flexion of $90^{\circ}$, with forearm supination. The surgical incision was made $2 \mathrm{~cm}$ lateral to the coracoid, was $5 \mathrm{~cm}$ long and was inferior along the edge of the deltoid muscle. The skin and subcutaneous tissue were subsequently cut to expose the cephalic vein. The cephalic vein was pulled to the medial, revealing the gap between the pectoralis major and the deltoid muscle. The long head tendon sheath of the biceps entered the cut to expose the broken LHB (sometimes, the ends of the LHB were under the pectoralis major tendon after rupture). After electrocoagulation of the anterior circumflex artery branch, along the distal extension line of the intertubercular sulcus and the upper edge of the pectoralis major tendon, a hole was drilled in the proximal humerus cortical bone, perpendicular to the medullary cavity, with a $4.0 \mathrm{~mm}$ drill, with the direction slightly towards the cephalad. A second hole was drilled with a $4.0 \mathrm{~mm}$ drill, $2 \mathrm{~cm}$ cranial to the first hole, with the direction slightly towards the caudal side. The broken end of the tendon was trimmed and the bicep tendon was tightened at the elbow flexion of $90^{\circ}$. Subsequently, the junction of the tendon and abdomen was at level with the lower edge of the pectoralis major tendon. The stump of the long head was removed parallel to the proximal bone hole. Two No. 5 Ethibond wires were used to knit the $2.5-\mathrm{cm}$ tendon ends (Fig. $1 \mathrm{~A}$ and B). The curved hollow guide pin was inserted from the lower hole to the upper hole, and the wire loop in the guide pin was extended to insert the folded No. 2 Ethibond wire. The curved hollow guide pin was removed, the blind end of the No. 2 Ethibond wire was removed from the hole of the distal cortical bone and the tendon-knitted wire was inserted into the distal blind end of the No. 2 Ethibond wire. The two free ends near the No. 2 Ethibond wire were pulled, the knitted wire was pulled into the medullary cavity from the lower bone hole and subsequently pulled out from the upper bone hole. The long-headed tendon was pulled into the medullary cavity from the distal bone hole by pulling the knitted wire and it was removed from the proximal end of the bone hole (Fig. 1C). The loop of the endobutton was subsequently cut off and the two ends of the two tendon-knitted wires were passed through the two holes at the center of the endobutton. The elbow was bent at $90^{\circ}$ and the forearm was rotated. The corresponding suture was knotted on the endobutton and the elbow joint was slightly stretched. The endobutton was then suspended from the surface near the bone hole and the LHB suspension fixation was complete (Fig. 1D). Post-operative bleeding was prevented following incision cleaning by suturing layer by layer. Antibiotics were routinely used $30 \mathrm{~min}$ prior to and $24 \mathrm{~h}$ following surgery.

Post-operative treatment. The upper limbs were suspended at $90^{\circ}$ for 6 weeks. Passive activity training began on the second day following surgery. Active elbow flexion was prohibited for 6 weeks and elbow weight training was prohibited for 12 weeks. Although passive activity training, including passive elbow flexion and extension, began on the second day following surgery.

Observation indexes and evaluation of therapeutic effect. Follow-up of patients was completed at 12 months following surgery. The visual analogue scale (VAS) score (7), elbow flexion strength (8), elbow joint supination muscle strength (9), the University of California Los Angeles (UCLA) shoulder score (10) and Rating Scale of American Shoulder Elbow Surgeons (ASES) score (11) were determined to evaluate the outcome of the surgery. These parameters were used to evaluate the discrepancies between pre-operation and post-operation.

Statistical analysis. SPSS 19 software (IBM Corp.) was used for statistical analysis. Values are expressed as the mean \pm standard deviation. Comparison between two groups (pre- and post-surgery) was performed using a student's t-test. $\mathrm{P}<0.05$ was considered to indicate a statistically significant difference.

\section{Results}

Surgery. Representative intra-operative images and a post-operative X-ray scanning image are presented in Fig. 2. Intraoperative bleeding was $30-50 \mathrm{ml}$. Gentle tissue separation reduced bleeding, and accurate incision positioning ensured moderate exposure of the LHB rupture end (Fig. 2A and B). The operation time was $\sim 40-70 \mathrm{~min}$.

Complications. After the surgery, the patients were followed up for one year. None of the 9 patients included in the present study exhibited any complications including nerve injury, infection and shoulder rigidity during or following the surgery. No pop-eye signs were observed during follow-up.

Surgical outcome at 1 year. At one year post-surgery, the VAS score, UCLA shoulder scores and ASES scores were significantly increased compared with the pre-operative values (Table I). All of the elbow flexion strength and elbow joint supination muscle strength were restored to grade $\mathrm{V}$ at the last follow-up. Active elbow flexion was allowed for 6 weeks after operation and elbow weight training was allowed for 12 weeks after operation. The upper limbs of all patients were fully weight-bearing at 6 months after operation.

\section{Discussion}

LHB lesions are a common cause of shoulder pain (12). LHB lesions are caused by an inflammatory reaction in or around the tendon or shoulder and may be due to instability or trauma (13). With developments in orthopedic science, the treatment for LHB lesions has been modified and improved. In 1940, LHB was considered to be the major source of shoulder pain, and due to this, tendon fixation was the first choice of treatment $(14,15)$. However, the current treatment options for LHB, which is frequently associated with pain, remain controversial. For refractory shoulder pain with secondary LHB lesions, the optimal treatment is surgery (16). However, the biggest drawback of LHB resection is that the tendon ends are free, exhibit a defective appearance and elbow strength may be reduced (17). Osbahr et al (18) suggested that tendon resection may be the cause of refractory and chronic biceps-derived pain.

LHB has recently been recognized as one of the causes of rotator cuff lesions and treatment using surgical LHB fixation 
Table I. Functional scores of patients with LHB rupture treated with the modified keyhole technique $(n=9)$.

\begin{tabular}{|c|c|c|c|c|c|}
\hline Evaluation & $\begin{array}{c}\text { Pain } \\
\text { (VAS scores) }\end{array}$ & $\begin{array}{l}\text { Elbow flexion } \\
\text { strength (grade) }\end{array}$ & $\begin{array}{l}\text { Strength of elbow } \\
\text { supination (grade) }\end{array}$ & UCLA scores & ASES scores \\
\hline Pre-operation & $7.22 \pm 1.20$ & 4 & 4 & $24.78 \pm 2.78$ & $73.22 \pm 3.63$ \\
\hline Post-operation (1 year) & $1.00 \pm 0.71$ & 5 & 5 & $31.89 \pm 2.26$ & $90.02 \pm 6.12$ \\
\hline t-value & 4.46 & & & 2.215 & 2.356 \\
\hline P-value & $<0.01$ & $<0.01$ & $<0.01$ & $<0.05$ & $<0.05$ \\
\hline
\end{tabular}

LHB, long head of biceps; UCLA, Shoulder Score of the University of California Los Angeles; ASES, Rating Scale of American Shoulder Elbow Surgeons.
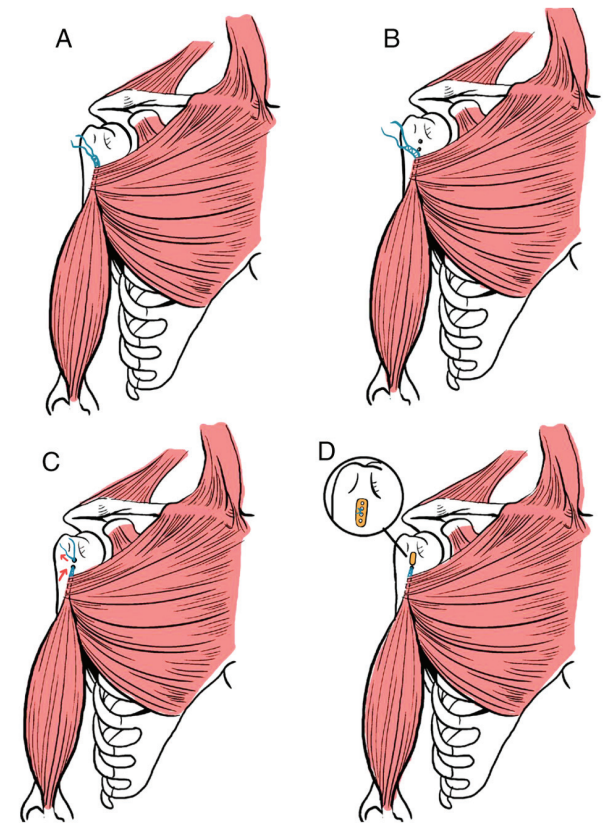

Figure 1. Schematic diagram of the surgical procedure. (A) Knit LHB end, (B) drilling of two holes above the pectoralis major tendon, (C) pull out LHB from the upper hole and (D) endobutton suspension and LHB fixation. LHB, long head of biceps.

has significantly expanded (19). Surgical indications of LHB fixation are tendon tear $(>50 \%)$, medial dislocation of the tendon and tearing of the subscapularis muscle combined with dislocation of LHB (20). Crenshaw and Kilgore (21) suggested that bicep-derived pain persisting for $>5$ months combined with limited activity indicates the requirement for surgical treatment.

LHB fixation may be performed using arthroscopy, via an open approach and via a combination of these methods. Numerous techniques for fixation have been demonstrated and four common types of surgical method exist for the treatment of LHB lesions: Bone tunnel, soft tissue tendon fixation, keyhole technique and anchor or interference screw fixation (5).

Soft tissue fixation and the keyhole technique are performed in an open surgery, whereas anchor and interference screw fixation are performed under arthroscopy. Amongst all surgical goals, the fixed strength is most important. The ideal fixation allows for active and passive activities of the

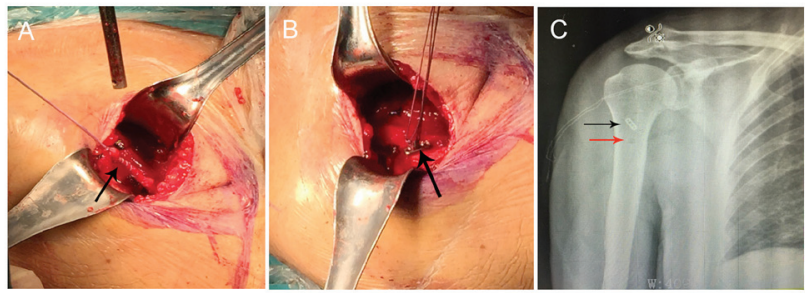

Figure 2. Case of a male patient (age, 65 years) with LHB rupture on the right side. (A) Knit LHB end during surgery (black arrow). (B) Reconstruction of the LHB on the extramedullary plate (black arrow). (C) Following surgery, the X-ray film reveals satisfactory internal fixation position (black arrow) and the lower hole (red arrow) is visible under the steel plate. LHB, long head of biceps.

shoulder joint in its full range, which is important for elderly patients and athletes due to the fact that a short shoulder joint fixation time may lead to adhesion of the shoulder joint and a decrease in muscle strength (22). The fixation strength of the interference screw technique is greater compared with that of the anchor, bone tunnel and keyhole techniques $(5,23,24)$. A cadaveric study by Buchholz et al (25) indicated that the use of intramedullary cortical bone plate to fix LHB exhibited no significant difference in static loading compared with the interference screw technique, but a decreased failure rate was observed in cyclic loading. Therefore, the intramedullary cortical plate has the best outcome in the treatment of LHB injuries.

Buchholz et al (25) also demonstrated that the intramedullary plate fixation technique by Siebenlist et al (6) uses a single-layer cortical bone suspension fixation, places the plate into the medullary canal and suspends the tendon in the extramedullary space. The compressive stress between the plate and the intracavitary surface of the cortical marrow leads to dissolving and absorption of cancellous bone osteolysis between the plate and the cortical bone surface of the medullary cavity, thereby resulting in fixation failure. Therefore, this single-cortical suspension in the medullary cavity is not reliable and the contralateral cortex requires to be perforated to pull the tendon, which may result in axillary nerve injury. Based on these effects, the keyhole technology was used in the present study for the treatment of LHB ruptures. Compared with the intramedullary fixation technique, two holes were drilled on the same side of the LHB and the endobutton was suspended outside the 
proximal cortical bone holes. In this technique, the osteolysis phenomenon may be reduced compared with the intramedullary suspension and the risk of axillary nerve injury may be avoided. In addition, the tendon was in contact with $\sim 2 \mathrm{~cm}$ of the surface cortical bone in the medullary cavity, which benefits tendon-bone healing. Compared with the traditional keyhole technique, the fixed strength of this technology is more reliable. However, in patients with LHB injury and rotator cuff tears, arthroscopic LHB was used with anchor fixation or tenotomy surgery.

Spatially, the LHB rupture surgery may be roughly divided into the rotator cuff interval, as well as the intra-articular and upper and lower edges of the pectoralis major tendon according to the fixed position. Fixation of LHB at the rotator cuff interval is frequently used when repairing the rotator cuff under arthroscopy. Intra-articular fixation of LHB may be performed using complete arthroscopy surgery and the fixation position may be at the humeral head cartilage margin, around the rotator cuff and at the apex of the intertubercular sulcus (26). Intra-articular fixation of LHB maximizes the original muscle tension of the biceps muscle and eliminates spasmodic pain and pop-eye sign. However, post-operative intractable pain has been previously reported following the proximal fixation of LHB, which was caused by extra-articular LHB tears and tenosynovitis in the intertubercular sulcus (27). A variety of scholars suggested that the 'hidden injury' located in the intertubercular sulcus is frequently missed under arthroscopy (28). Therefore, LHB is fixed at a low position to prevent pain in the extra-articular and intertubercular sulcus and to eliminate the cause of post-operative persistent pain.

For simple SLAP injuries, intra-articular fixation of LHB is a useful alternative surgical treatment (5). However, for other LHB tendon disorders, including LHB ruptures in the intertubercular sulcus, which occurs in the majority of cases due to the friction experienced by the intertubercular sulcus, fixation in the intertubercular sulcus or upper edge of the pectoralis major tendon is more effective (28). For most patients with LHB rupture, the tendon rupture occurs in the intertubercular sulcus, and the preferred method of repair at our institution is fixation under the intertubercular sulcus and upper the pectoralis major tendon (5). The advantages of the technique presented in the present study are that the tendon injury derived from the intertubercular sulcus, which are often ignored by surgeons, may be eliminated and adequate biceps muscular tension is maintained.

Based on the strict selection criterion that the patients had isolated LHB rupture, the present study examined a limited number of cases with a relatively short follow-up period. Therefore, it was not possible to include a control group. The reasons for the lack of post-operative complications observed were the limited number of patients and simple operation technique. For the cases of isolated LHB rupture, a modified keyhole surgical technique was used and combined with microplate suspension to treat the LHB tendon. This treatment has the advantages of low difficulty in operation, high safety and a resultant reliable fixation.

The results of the present study demonstrated that with keyhole technology, the intramedullary microplate is a reliable fixation treatment for LHB rupture.

\section{Acknowledgements}

Not applicable.

\section{Funding}

No funding was received.

\section{Availability of data and materials}

The datasets used and/or analyzed during the present study are available from the corresponding author on reasonable request.

\section{Authors' contributions}

YH wrote this manuscript. YH, LNS, HS and BH collected and analyzed the data. YH and LNS performed the experiments. FCZ, CSZ and CJP interpreted the results. LNS revised and finalized the study. All authors read and approved the final manuscript.

\section{Ethics approval and consent to participate}

The present study was approved by the Ethics Committee of the Affiliated Hospital of Nanjing University of Chinese Medicine (Nanjing, China). The patients who participated in this study had complete clinical data. Informed consent was obtained from the patients or their guardians.

\section{Patient consent for publication}

Not applicable.

\section{Competing interests}

The authors declare that they have no competing interests.

\section{References}

1. Alpantaki K, McLaughlin D, Karagogeos D, Hadjipavlou A and Kontakis G: Sympathetic and sensory neural elements in the tendon of the long head of the biceps. J Bone Joint Surg Am 87: 1580-1583, 2005.

2. Elser F, Braun S, Dewing CB, Giphart JE and Millett PJ: Anatomy, function, injuries, and treatment of the long head of the biceps brachii tendon. Arthroscopy 27: 581-592, 2011.

3. Gaskill TR, Braun S and Millett PJ: Multimedia article. The rotator interval: Pathology and management. Arthroscopy 27: 556-567, 2011.

4. Frost A, Zafar MS and Maffulli N: Tenotomy versus tenodesis in the management of pathologic lesions of the tendon of the long head of the biceps brachii. Am J Sports Med 37: 828-833, 2009.

5. Mazzocca AD, Bicos J, Santangelo S, Romeo AA and Arciero RA: The biomechanical evaluation of four fixation techniques for proximal biceps tenodesis. Arthroscopy 21: 1296-1306, 2005.

6. Siebenlist S, Lenich A, Buchholz A, Martetschläger F, Eichhorn S, Heinrich P, Fingerle A, Doebele S, Sandmann GH, Millett PJ, et al: Biomechanical in vitro validation of intramedullary cortical button fixation for distal biceps tendon repair: A new technique. Am J Sports Med 39: 1762-1768, 2011.

7. Lopiz Y, Alcobía-Díaz B, Galán-Olleros M, García-Fernández C, Picado AL and Marco F: Reverse shoulder arthroplasty versus nonoperative treatment for 3- or 4-part proximal humeral fractures in elderly patients: A prospective randomized controlled trial. J Shoulder Elbow Surg: Sep 6, 2019 doi: 10.1016/j. jse.2019.06.024 (Epub ahead of print). 
8. Frank T, Seltser A, Grewal R, King GJW and Athwal GS Management of chronic distal biceps tendon ruptures: Primary repair vs. semitendinosus autograft reconstruction. J Shoulder Elbow Surg 28: 1104-1110, 2019.

9. Sarda P, Qaddori A, Nauschutz F, Boulton L, Nanda R and Bayliss N: Distal biceps tendon rupture: Current concepts. Injury 44: 417-420, 2013.

10. Rhee SM, Jeong HY, Ro K, Pancholi S and Rhee YG: Double on-lay fixation using all suture-type anchor for subpectoral biceps tenodesis has favorable functional outcomes and leads to less cosmetic deformities than single on-lay fixation. Knee Surg Sports Traumatol Arthrosc: Aug 13, 2019 doi: 10.1007/s00167-019-05663-4 (Epub ahead of print).

11. Brochin RL, Zastrow R, Hussey-Andersen L, Parsons BO and Cagle PJ: Revision rotator cuff repair: A systematic review. J Shoulder Elbow Surg: Aug 28, 2019 doi: 10.1016/j. jse.2019.06.023 (Epub ahead of print)

12. Urita A, Funakoshi T, Amano T, Matsui Y, Kawamura D, Kameda Y and Iwasaki N: Predictive factors of long head of the biceps tendon disorders-the bicipital groove morphology and subscapularis tendon tear. J Shoulder Elbow Surg 25: 384-389, 2016.

13. Zabrzynski J, Paczesny L, Lapaj L, Grzanka D and Szukalski J: Process of neovascularization compared with pain intensity in tendinopathy of the long head of the biceps brachii tendon associated with concomitant shoulder disorders, after arthroscopic treatment. Microscopic evaluation supported by immunohistochemical. Folia Morphol (Warsz) 77: 378-385, 2018.

14. Depalma AF and Callery GE: Bicipital tenosynovitis. Clin Orthop 3: 69-85, 1954.

15. Yedinak PR and Holbrook BG: Bicipital tenosynovitis. Rocky Mt Med J 51: 185-191, 1954.

16. Bhatia DN, van Rooyen KS and de Beer JF: Direct arthroscopy of the bicipital groove: A new approach to evaluation and treatment of bicipital groove and biceps tendon pathology. Arthroscopy 24: 368.e1-e6, 2008

17. Galasso O, Gasparini G, De Benedetto M, Familiari F and Castricini R: Tenotomy versus tenodesis in the treatment of the long head of biceps brachii tendon lesions. BMC Musculoskelet Disord 13: 205, 2012.

18. Osbahr DC, Diamond AB and Speer KP: The cosmetic appearance of the biceps muscle after long-head tenotomy versus tenodesis. Arthroscopy 18: 483-487, 2002.
19. Kane P, Hsaio P, Tucker B and Freedman KB: Open subpectoral biceps tenodesis: Reliable treatment for all biceps tendon pathology. Orthopedics 38: 37-41, 2015.

20. Lo IK and Burkhart SS: Arthroscopic biceps tenodesis using a bioabsorbable interference screw. Arthroscopy 20: 85-95, 2004.

21. Crenshaw AH and Kilgore WE: Surgical treatment of bicipital tenosynovitis. J Bone Joint Surg Am 48: 1496-1502, 1966.

22. Eakin CL, Faber KJ, Hawkins RJ and Hovis WD: Biceps tendon disorders in athletes. J Am Acad Orthop Surg 7: 300-310, 1999.

23. Golish SR, Caldwell PE III, Miller MD, Singanamala N, Ranawat AS, Treme G, Pearson SE, Costic R and Sekiya JK: Interference screw versus suture anchor fixation for subpectoral tenodesis of the proximal biceps tendon: A cadaveric study. Arthroscopy 24: 1103-1108, 2008.

24. Ozalay M, Akpinar S, Karaeminogullari O, Balcik C, Tasci A, Tandogan RN and Gecit R: Mechanical strength of four different biceps tenodesis techniques. Arthroscopy 21: 992-998, 2005.

25. Buchholz A, Martetschlager F, Siebenlist S, Sandmann GH, Hapfelmeier A, Lenich A, Millett PJ, Stöckle U and Elser F: Biomechanical comparison of intramedullary cortical button fixation and interference screw technique for subpectoral biceps tenodesis. Arthroscopy 29: 845-853, 2013.

26. Elkousy HA, Fluhme DJ, O'Connor DP and Rodosky MW: Arthroscopic biceps tenodesis using the percutaneous, intra-articular trans-tendon technique: Preliminary results. Orthopedics 28: 1316-1319, 2005

27. Becker DA and Cofield RH: Tenodesis of the long head of the biceps brachii for chronic bicipital tendinitis. Long-term results. J Bone Joint Surg Am 71: 376-381, 1989.

28. Moon SC, Cho NS and Rhee YG: Analysis of 'hidden lesions' of the extra-articular biceps after subpectoral biceps tenodesis: The subpectoral portion as the optimal tenodesis site. Am J Sports Med 43: 63-68, 2015.

This work is licensed under a Creative Commons Attribution-NonCommercial-NoDerivatives 4.0 International (CC BY-NC-ND 4.0) License. 